\title{
EXPONENTIAL DECAY IN NONLINEAR NETWORKS ${ }^{1}$
}

\author{
R. J. DUFFIN
}

1. Introduction. This paper is concerned with the effect of friction or damping on electrical or mechanical systems. Of particular concern is the rate of decay of the amplitude of free vibrations. For systems described by linear differential equations with constant coeffcients, it is well known that damping causes the free vibrations to decay exponentially. It is to be shown here that a similar behavior holds for a class of nonlinear systems.

To clarify the mathematical formulation of the problem, attention will first be confined to a mechanical system having only one degree of freedom. Let the Lagrangian coordinate be denoted by $q$ and let $\dot{q}=d q / d t$ denote the velocity. The Lagrangian equation for the system may be written as

$$
d p_{1} / d t+p_{2}+p_{3}=g \text {. }
$$

Here $g$ is interpreted as the external force applied to the system, $d p_{1} / d t$ is the inertial force, $p_{2}$ is the damping force, and $p_{3}$ is the elastic force. The free vibrations are determined by taking $g=0$.

If the system is linear $p_{1}=L \dot{q}, p_{2}=R \dot{q}$, and $p_{3}=S q$ where $L, R$, and $S$ are constants which satisfy $L \geqq 0, S \geqq 0$, and $R>0$. Thus the free vibration of a linear system is determined by the equation

$$
L \ddot{q}+R \dot{q}+S q=0 .
$$

This equation can be solved explicitly and it is seen that $\dot{q}=O\left(e^{-b t}\right)$ for some positive constant $b$. Thus the velocity decays exponentially.

The nonlinear systems under consideration are assumed to satisfy the relations $p_{1}=V_{1}(\dot{q}), p_{2}=R \dot{q}$, and $p_{3}=V_{3}(q)$. Here $V_{1}(\dot{q})$ is a function only of $\dot{q}$ and $V_{3}(q)$ is a function only of $q$. It is further assumed that $0=V_{1}(0)=V_{3}(0)$ and that there are positive constants $A$ and $B$ such that:

$$
A \leqq d V_{1}(x) / d x \leqq B
$$

and

$$
A \leqq d V_{3}(x) / d x \leqq B \text { for all } x \text {. }
$$

Received by the editors October 19, 1953 and, in revised form, December 22, 1955.

1 The work on this paper was sponsored by the Office of Ordnance Research, U. S. Army, Contract No. DA-36-061-ORD-378. 
$R$ is permitted to be an arbitrary variable but subject to the condition

$$
A \leqq R \leqq B .
$$

In particular $R$ could be a function of $q, \dot{q}$, and $t$. The same constants $A$ and $B$ appear in relations (b), (c), and (d) only as a matter of convenience in notation. The Lagrangian equation becomes

$$
d V_{1}(\dot{q}) / d t+R \dot{q}+V_{3}(q)=0 .
$$

The hypotheses made above are sufficiently general to insure that a large class of physical systems are described by an equation of the type $\left(a^{\prime \prime}\right)$.

To prove that the solutions of $\left(a^{\prime \prime}\right)$ decay exponentially it is first observed that (b) or (c) imply

$$
A \leqq V(x) / x \leqq B .
$$

(Here the subscript has been dropped.) Let $v(x)=\int_{0}^{x} V(x) d x$, then it follows from (e) that

$$
A x^{2} \leqq 2 v(x) \leqq B x^{2} .
$$

Let $w(x)=\int_{0}^{x} x d V(x)$, then it follows from (b) or (c) that

$$
A x^{2} \leqq 2 w(x) \leqq B x^{2} .
$$

Multiplying $\left(\mathrm{a}^{\prime \prime}\right)$ by $\dot{q}$ gives

$$
\dot{q} d V_{1}(\dot{q}) / d t+V_{3}(q) \dot{q}=-R \dot{q}^{2}
$$

or

$$
d\left(w_{1}(\dot{q})+v_{3}(q)\right) / d t=-R \dot{q}^{2} .
$$

(It is of interest to observe that $w_{1}(\dot{q})$ can be interpreted as the kinetic energy of the system and $v_{3}(q)$ can be interpreted as the potential energy.) It also follows from $\left(a^{\prime \prime}\right)$ that

$$
d\left(q V_{1}(\dot{q})\right) / d t=\dot{q} V_{1}(\dot{q})-R \dot{q} q-V_{3}(q) q .
$$

Let $f=w_{1}+v_{3}+b q V_{1}$ and $F=-\dot{f}-b f$ where $b$ is a positive constant. Then

$$
F=R \dot{q}^{2}-b \dot{q} V_{1}+b R \dot{q} q+b V_{3} q-b w_{1}-b v_{3}-b^{2} V_{1} q .
$$

Since $w_{3}(q)+v_{3}(q)=V_{3}(q) q$ it follows that

$$
F=R \dot{q}^{2}-b \dot{q} V_{1}-b w_{1}+b R \dot{q} q-b^{2} V_{1} q+b w_{3} .
$$

Making use of inequalities (e), (f), and (g) gives

$$
F \geqq(A-3 b B / 2) \dot{q}^{2}-B\left(b+b^{2}\right)|\dot{q}||q|+b A q^{2} / 2 .
$$


The expression is a quadratic form in $|q|$ and $|\dot{q}|$; it is non-negative if

$$
4(\rho-3 b / 2)(\rho b / 2)-\left(b+b^{2}\right)^{2} \geqq 0, \quad \rho=A / B .
$$

Let $b$ have the value $\rho^{2} / 4$, then this last relation becomes

$$
\rho^{4}(1-3 \rho / 8) / 2-\rho^{4}\left(1 / 4+\rho^{2} / 16\right)^{2}>\rho^{4} / 16>0 .
$$

Thus $F \geqq 0$ or equivalently $d\left(e^{b t} f\right) / d t \leqq 0$. From the definition of $f$ and the inequalities (e), (f), and (g) it follows that

$$
f \geqq A \dot{q}^{2} / 2+A q^{2} / 2-b B|\dot{q}||q| \text {. }
$$

Since $b B=\rho A / 4 \leqq A / 4$ it is seen that

$$
f \geqq A\left(\dot{q}^{2}+q^{2}\right) / 4 \text {. }
$$

Thus $f$ is non-negative so the relation $d\left(e^{b t} f\right) / d t \leqq 0$ implies that $f=O\left(e^{-b t}\right)$. Thus $q^{2}=O\left(e^{-b t}\right)$ and $\dot{q}^{2}=O\left(e^{-b t}\right)$. This completes the proof that all solutions of the differential equation $\left(a^{\prime \prime}\right)$ vanish exponentially.

Now consider mechanical systems with $n$ degrees of freedom. By use of vectorial notation the Lagrangian equations may be expressed as a single vector equation of the form (a). Now $p_{1}, p_{2}, p_{3}$, and $g$ denote vectors with $n$ components. If the system is linear then the free vibrations are determined by equation (a) where $L, R$, and $S$ signify $n$ by $n$ matrices and $q$ is a vector with $n$ components. In the "normal case" the matrices $L, R$, and $S$ are constant, symmetric, and semi-definite. If $R$ is positive definite, standard techniques may be applied to show that all solutions vanish exponentially.

The nonlinear systems to be considered are governed by a vector equation of the form $\left(\mathrm{a}^{\prime \prime}\right)$. Now $R$ denotes a variable, positive, definite matrix. The expressions $V_{1}$ and $V_{3}$ are nonlinear transformations of a type which are here termed "definite transformations." The properties of definite transformations are developed in $\$ 2$.

Of concern in $\$ 2$ are transformations $V(q)=p$ where $p$ and $q$ are vectors. If $q_{1}, q_{2}, \cdots, q_{n}$ denote the coordinates of $q$, then the coordinates of $p$ are given by

$$
\begin{gathered}
p_{1}=V_{1}\left(q_{1}, q_{2}, \cdots, q_{n}\right), \\
p_{2}=V_{2}\left(q_{1}, q_{2}, \cdots, q_{n}\right), \\
\cdot \cdot \cdot \cdot \cdot \cdot \cdot, \\
p_{n}=V_{n}\left(q_{1}, q_{2}, \cdots, q_{n}\right),
\end{gathered}
$$

The Jacobian matrix of this transformation is 


$$
V_{i j}^{\prime}=\partial V_{i} / \partial q_{j}, \quad i, j=1,2, \cdots n \text {. }
$$

It is assumed that this matrix is symmetric, thus $V_{i j}^{\prime}=V_{j l}^{\prime}$. It is further assumed that the origin is a fixed point of the transformation. Then the transformation $V$ is termed strictly definite, if there are positive constants $A$ and $B$ such that the eigenvalues $\lambda_{i}$ of the matrix $V_{i j}^{\prime}$ satisfy $A \leqq \lambda_{i} \leqq B$. The transformation is termed definite if some of the eigenvalues satisfy this inequality while the others vanish. In general, the eigenvalues will be functions of $q$. It is seen that in the linear case a strictly definite transformation has a positive definite matrix and a definite transformation has a semi-definite matrix.

The proof of exponential decay for systems with $n$ degrees of freedom is given in $\$ 3$. As in the special case just considered a scalar function $f(q, \dot{q})$ is introduced which is shown to satisfy the inequalities $f \geqq 0$ and $d f / d t+b f \leqq 0$. It follows that $f=O\left(e^{-b t}\right)$ and from this it is seen that $\dot{q}$ decays exponentially.

The proof of exponential decay is based on the hypothesis that $R$ is positive definite. In physical terms this hypothesis might be described as "distributed damping." It is possible to give examples of systems which have exponential decay but with $R$ merely semi-definite. Some results for systems in which $R$ is merely semi-definite have been obtained in a previous paper [1].

The last section of this paper is concerned with nonlinear electrical networks. It is pointed out that the current $\dot{q}$ flowing in a network is governed by an equation of the form (a). The theorems proved in this paper are shown to impose certain restrictions on the behavior of nonlinear networks.

2. Definite transformations and definite forms. Let $Q$ be a real $n$-dimensional vector space. If $p$ and $q$ are vectors of $Q$, let $(p, q)$ denote the scalar product and let $\|q\|=(q, q)^{1 / 2}$ denote the norm.

An $n$ by $n$ matrix $L$ defines a linear transformation of $Q$, say $p=L q$. If $L$ is symmetric and $(L q, q) \geqq 0$, then $L$ is said to be definite. If $(L q, q)>0$ for $q \neq 0$, then $L$ is said to be strictly definite. (This terminology is slightly unorthodox.)

The notion of a definite matrix is of prime importance in the study of linear networks. In nonlinear networks the linear transformation $L q$ is replaced by a nonlinear transformation $V(q)$. In many cases of physical importance it results that $V(q)$ has properties quite similar to $L q$. This was shown in previous papers, and $V(q)$ was termed a quasi-linear replacement of $L q$. The theory of such nonlinear transformations is given here in a somewhat more systematic and complete form than in the previous papers $[1 ; 2 ; 3]$. 
Let $p=V(q)$ be a continuous transformation of $Q$ defined for every vector $q$. Let $d p=V^{\prime} d q$ denote the corresponding differential transformation. The matrix $V^{\prime}$ is supposed to have matrix elements which are continuous functions of $q$. This transformation $p=V(q)$ is termed definite if:

(i) $V(0)=0$;

(ii) $V^{\prime}$ is symmetric;

(iii) $A\left\|x_{1}\right\|^{2} \leqq\left(V^{\prime} x, x\right) \leqq B\left\|x_{1}\right\|^{2}$.

Here $x_{1}$ denotes the orthogonal projection of the arbitrary vector $x$ in a subspace $Q_{1}$ of $Q$. $A$ and $B$ are positive constants termed bounds. $Q_{1}$ depends on $V$. If $Q_{1}$ can be taken to be $Q$, then the transformation is termed strictly definite.

It may be seen that this definition is so phrased that if $L$ is a definite matrix, then $p=L q$ is a definite transformation. The following lemmas pertain to a definite transformation $p=V(q)$.

LEMmA 1. With each pair of vectors $q^{\prime}$ and $q^{\prime \prime}$ there is associated a definite matrix $V^{*}$ such that

$$
V\left(q^{\prime}\right)-V\left(q^{\prime \prime}\right)=V^{*} q^{\prime}-V^{*} q^{\prime \prime}
$$

and

$$
A\left\|x_{1}\right\|^{2} \leqq\left(V^{*} x, x\right) \leqq B\left\|x_{1}\right\|^{2}
$$

for an arbitrary vector $x$.

Proof. Let $q=q^{\prime \prime}+s\left(q^{\prime}-q^{\prime \prime}\right)$ where $s$ is a parameter. Then $d q=d s\left(q^{\prime}-q^{\prime \prime}\right)$ and

$$
p^{\prime}-p^{\prime \prime}=\int d p=\int_{0}^{1} V^{\prime} d s\left(q^{\prime}-q^{\prime \prime}\right) .
$$

This proves (1) with

$$
V^{*}=\int_{0}^{1} V^{\prime} d s .
$$

Integrating (iii) with respect to $s$ gives (2). (In previous papers $V^{*}$ was also denoted as $V^{\prime}$.)

A consequence of (2) is

$$
A\left\|x_{1}\right\| \leqq\left\|V^{*} x\right\| \leqq B\left\|x_{1}\right\| .
$$

This may be seen by considering a rotated coordinate system in which $V^{*}$ is in diagonal form.

Taking $q^{\prime}=q$ and $q^{\prime \prime}=0$ gives 


$$
V(a)=V^{*} q .
$$

Then by (4)

$$
A\left\|q_{1}\right\| \leqq\|V(q)\| \leqq B\left\|q_{1}\right\| .
$$

Lemma 2. $V\left(q^{\prime}\right)=V\left(q^{\prime \prime}\right)$ if and only if the projection of $q^{\prime}-q^{\prime \prime}$ in $Q_{1}$ vanishes.

Proof. This follows from (1) and (4) by taking $x=q^{\prime}-q^{\prime \prime}$.

LEMma 3. The scalar function

$$
v(q)=\int_{0}^{1}(V(s q), q) d s
$$

is the potential of $V$. That is

$$
d v(q)=(V(q), d q) .
$$

Moreover,

$$
A\left\|q_{1}\right\|^{2} \leqq 2 v(q) \leqq B\left\|q_{1}\right\|^{2} .
$$

Proof. Differentiating (7) gives

$$
\begin{aligned}
d v & =\int_{0}^{1}\left[\left(s V^{\prime} d q, q\right)+(V(s q), d q)\right] d s \\
& =\int_{0}^{1}\left(s V^{\prime} q+V(s q), d q\right) d s \\
& =\int_{0}^{1}(d V(s q) s / d s, d q) d s=(V(q), d q) .
\end{aligned}
$$

In Lemma 1 take $q^{\prime}=s q$ and $q^{\prime \prime}=0$. Then $V(s q)=V^{*} s q$ and $(V(s q), q)$ $=\left(V^{*} q, q\right) s$. Integrating (2) with respect to $s$ proves (9). The potential of a definite transformation may be termed a definite form.

Lemma 4. The range of $V(q)$ is precisely $Q_{1}$.

Proof. Relation (6) implies that the range of $V(q)$ is contained in $Q_{1}$. This result, together with Lemma 2, shows that it is sufficient to confine attention to vectors in the subspace $Q_{1}$. Let $p^{\prime}$ be a given vector of $Q_{1}$. Let $u=v(q)-\left(p^{\prime}, q\right)$ for $q$ in $Q_{1}$. Then by (9) it is seen that $u \rightarrow+\infty$ as $\|q\| \rightarrow \infty$. Hence $u$ has an absolute minimum at some point, say $q=q^{\prime}$. Thus

$$
0=d u=d v-\left(p^{\prime}, d q\right)=\left(V\left(q^{\prime}\right)-p^{\prime}, d q\right) .
$$


It follows that $V\left(q^{\prime}\right)-p^{\prime}=0$. Since $p^{\prime}$ is arbitrary, this completes the proof.

LEмма 5. There is a definite transformation $W$ whose range is $Q_{1}$ and such that $W$ and $V$ are inverse transformations in $Q_{1}$.

Proof. Attention is first confined to the subspace $Q_{1}$. By Lemma 2 and Lemma 4 it follows that $V$ has a unique inverse, say $q=W(p)$. The Jacobian of the transformation $p=V(q)$ is the determinant of $V^{\prime}$; this does not vanish. Thus by a standard theorem of the differential calculus $d q=W^{\prime} d p$ and the matrix elements of $W^{\prime}$ are continuous functions of $p$. Since the matrix $W^{\prime}$ is the inverse of $V^{\prime}$ it follows that $W^{\prime}$ is symmetric. Moreover,

$$
B^{-1}\|x\|^{2} \leqq\left(W^{\prime} x, x\right) \leqq A^{-1}\|x\|^{2}
$$

for any vector $x$ of $Q_{1}$. This is seen by taking a rotated coordinate system in which $V^{\prime}$ is in diagonal form. Relation (10) shows that $W$ satisfies condition (iii). Thus $W$ is a strictly definite transformation of $Q_{1}$.

For any vector $p$ of $Q$ define $W(p)=W\left(p_{1}\right)$ where $p_{1}$ is the projection of $p$ in $Q_{1}$. It is clear that $W$ so defined is a definite transformation of $Q$.

Lemma 6. Let $p=V(q)$. Then the potentials of $V$ and $W$ are related by

$$
v(q)+w(p)=(p, q) \text {. }
$$

Moreover,

$$
A\left\|q_{1}\right\|^{2} \leqq 2 w(p) \leqq B\left\|q_{1}\right\|^{2} .
$$

Proof. Let $h=v+w-(p, q)$. Then

$$
d h=d v-(p, d q)+d w-(q, d p)=d w-(q, d p) .
$$

But $W(p)=q_{1}$ and $d w=\left(q_{1}, d p\right)=(q, d p)$. The last step is a consequence of $d p$ being in $Q_{1}$. Thus $d h=0$, so $h$ is constant. But if $q=0$, then $h=0$, so (11) follows.

By relation (7)

$$
\begin{aligned}
w(p) & =(V(q), q)-\int_{0}^{1}(V(s q), q) d s \\
& =\int_{0}^{1}(V(q)-V(s q), q) d s=\int_{0}^{1}\left(V^{*} q, q\right)(1-s) d s .
\end{aligned}
$$

Integrating inequality (2) proves (12). 
Lemma 7. Let $k$ be a constant vector. Then $V(q+k)-V(k)$ is a definite transformation with the same range as $V(q)$.

Proof. It is obvious that this transformation satisfies the conditions (i), (ii), and (iii).

3. Exponential decay. A vector $q$ in the space $Q$ is assumed to vary with time so as to satisfy a differential equation of the form

$$
d p_{1} / d t+p_{2}+p_{3}=g .
$$

It is assumed that the first derivatives of the vectors $p_{1}$ and $q$ exist for $t \geqq 0$. Equation (13) contains definite transformations. Unless the contrary is stated, these are considered not to vary with the time.

If a function is $O\left(e^{-c t}\right)$ for some positive constant $c$, it is said to vanish exponentially.

Theorem 1. Let $V_{1}$ and $V_{3}$ be definite transformations. In the differential equation

$$
d V_{1}(\dot{q}) / d t+R \dot{q}+V_{3}(q)=0
$$

$R$ denotes a matrix whose matrix elements may depend on $t, q$, and $\dot{q}$. It is supposed that there are positive constants $A$ and $B$ such that

$$
A\|x\|^{2} \leqq(R x, x) \text { and }\|R x\| \leqq B\|x\|
$$

for an arbitrary vector $x$. Let $q_{3}$ denote the projection of $q$ in the subspace which is the range of $V_{3}$.

Then there are positive constants $a$ and $b$ such that for all solutions of (14)

$$
e^{-a t} \leqq\|\dot{q}\|^{2}+\left\|q_{3}\right\|^{2} \leqq e^{-b t}
$$

provided $t$ is sufficiently large and $\dot{q}$ does not vanish identically.

Proof. Comparing (13) and (14) gives $p_{1}=V_{1}(\dot{q}), p_{2}=R \dot{q}$, and $p_{3}=V_{3}(q)$. Let $\dot{q}_{1}$ denote the projection of $\dot{q}$ in $Q_{1}$, the range of $V_{1}$. It is not assumed that $R$ is symmetric.

By (8), $d v_{3}(q) / d t=\left(V_{3}(q), \dot{q}\right)=\left(p_{3}, \dot{q}\right)$. By $(11), d w_{1}\left(p_{1}\right)=\left(\dot{q}, d p_{1}\right)$, so $d w_{1}\left(p_{1}\right) / d t=\left(\dot{q}, d p_{1} / d t\right)$. Thus

$$
d\left(w_{1}+v_{3}\right) / d t=-\left(p_{2}, \dot{q}\right)=-(R \dot{q}, \dot{q}) .
$$

Let the form $f$ be defined as

$$
f=w_{1}+v_{3}+b\left(p_{1}, q_{3}\right)
$$

where $b$ is a positive constant. Then 


$$
d\left(p_{1}, q_{3}\right) / d t=\left(\dot{p}_{1}, q_{3}\right)+\left(p_{1}, \dot{q}_{3}\right)=-\left(p_{2}, q_{3}\right)-\left(p_{3}, q_{3}\right)+\left(p_{1}, \dot{q}_{3}\right) .
$$

Making use of this relation together with (17) gives

$$
\dot{f}=-\left(p_{2}, \dot{q}\right)+b\left(p_{1}, \dot{q}_{3}\right)-b\left(p_{2}, q_{3}\right)-b\left(p_{3}, q_{3}\right) .
$$

Let $F=-\dot{f}-b f$. Then

$$
F=\left(p_{2}, \dot{q}\right)-b w_{1}-b\left(p_{1}, \dot{q}_{3}\right)+b\left(p_{2}, q_{3}\right)-b^{2}\left(p_{1}, q_{3}\right)+b w_{3} .
$$

Here relation (11) has been employed; that is, $w_{3}=\left(p_{3}, q_{3}\right)-v_{3}$.

Without loss of generality it may be assumed that $A$ and $B$ in (15) are also bounds for the definite transformations $V_{1}$ and $V_{3}$. Then by the inequalities (15) together with those given in $\$ 2$ :

$$
\begin{aligned}
\left(p_{2}, \dot{q}\right) & =(R \dot{q}, \dot{q}) \geqq A\|\dot{q}\|^{2}, \\
w_{3} & \geqq A\left\|q_{3}\right\|^{2} / 2, \\
w_{1} & \leqq B\left\|\dot{q}_{1}\right\|^{2} / 2 \leqq B\|\dot{q}\|^{2} / 2, \\
\left|\left(p_{1}, \dot{q}_{3}\right)\right| & \leqq\left\|p_{1}\right\|\left\|\dot{q}_{3}\right\| \leqq B\|\dot{q}\|^{2}, \\
\left|\left(p_{2}, q_{3}\right)\right| & \leqq\left\|p_{2}\right\|\left\|q_{3}\right\| \leqq B\|\dot{q}\|\left\|q_{3}\right\|,
\end{aligned}
$$

and

$$
\left|\left(p_{1}, q_{3}\right)\right| \leqq\left\|p_{1}\right\|\left\|q_{3}\right\| \leqq B\|\dot{q}\|\left\|q_{3}\right\| .
$$

Substituting these inequalities in the expression for $F$ gives

$$
F \geqq(A-3 b B / 2)\|\dot{q}\|^{2}-B\left(b+b^{2}\right)\|\dot{q}\|\left\|q_{3}\right\|+b A\left\|q_{3}\right\|^{2} / 2 .
$$

The expression on the right is non-negative if

$$
4(\rho-3 b / 2)(\rho b / 2)-\left(b+b^{2}\right)^{2} \geqq 0, \quad \rho=A / B .
$$

Take $b=\rho^{2} / 4$. Then this relation becomes

$$
\rho^{4}(1-3 \rho / 8) / 2-\rho^{4}\left(1 / 4+\rho^{2} / 16\right)^{2}>\rho^{4} / 16>0 .
$$

Thus $F \geqq 0$ or equivalently $d\left(e^{b t} f\right) / d t \leqq 0$. Hence, for $t \geqq 0, f(0) \geqq e^{b t} f(t)$ by the law of the mean, so

$$
f(t) \leqq f(0) e^{-b t}, \quad b=A^{2} / 4 B^{2} .
$$

Clearly $f \geqq A\left\|\dot{q}_{1}\right\|^{2} / 2+A\left\|q_{3}\right\|^{2} / 2-b B\left\|\dot{q}_{1}\right\|\left\|q_{3}\right\|$. Substituting the value of $b$ stated in (19) gives $b B \leqq A / 4$, so

$$
f \geqq(A / 4)\left(\left\|\dot{q}_{1}\right\|^{2}+\left\|q_{3}\right\|^{2}\right) .
$$

Let $\dot{q}=\dot{q}_{1}+\dot{q}_{0}$ where $\dot{q}_{0}$ is in the orthogonal complement of $Q_{1}$. Then since $d p_{1} / d t$ is in $Q_{1}$, it follows from (14) that $\left(R \dot{q}, \dot{q}_{0}\right)+\left(p_{3}, \dot{q}_{0}\right)=0$. Thus 


$$
\begin{aligned}
\left(R \dot{q}_{0}, \dot{q}_{0}\right) & =-\left(R \dot{q}_{1}+p_{3}, \dot{q}_{0}\right) \leqq\left\|R \dot{q}_{1}+p_{3}\right\|\left\|\dot{q}_{0}\right\|, \\
A\left\|\dot{q}_{0}\right\| & \leqq\left\|R \dot{q}_{1}+p_{3}\right\| \leqq\left\|R \dot{q}_{1}\right\|+\left\|p_{3}\right\|, \\
\left\|\dot{q}_{0}\right\| & \leqq(B / A)\left(\left\|\dot{q}_{1}\right\|+\left\|q_{3}\right\|\right) \\
\left\|\dot{q}_{0}\right\|^{2} & \leqq 2(B / A)^{2}\left(\left\|\dot{q}_{1}\right\|^{2}+\left\|q_{3}\right\|^{2}\right) .
\end{aligned}
$$

Since $\|\dot{q}\|^{2}=\left\|\dot{q}_{1}\right\|^{2}+\left\|\dot{q}_{0}\right\|^{2}$

$$
\|\dot{q}\|^{2}+\left\|q^{3}\right\|^{2} \leqq\left(1+2(B / A)^{2}\right)\left(\left\|\dot{q}_{1}\right\|^{2}+\left\|q_{3}\right\|^{2}\right) .
$$

From (19), (20), and (21) it follows that $\|\dot{q}\|^{2}+\left\|q_{3}\right\|^{2}=O\left(e^{-b t}\right)$. It is clear from the derivation that a slightly larger value of $b$ could have been chosen. This proves one part of (16).

Let $E=w_{1}+v_{3}$, then

$$
\dot{E}+a E=-\left(p_{2}, \dot{q}\right)+a E \geqq-B\|\dot{q}\|^{2}+a A\left(\left\|\dot{q}_{1}\right\|^{2}+\left\|q_{3}\right\|^{2}\right) / 2 .
$$

Making use of (21) gives

$$
\dot{E}+a E \geqq\left(-B\left[1+2(B / A)^{2}\right]+a A / 2\right)\left(\left\|\dot{q}_{1}\right\|^{2}+\left\|q_{3}\right\|^{2}\right) .
$$

So if $a \geqq 6(B / A)^{3}$, then $\dot{E}+a E \geqq 0, d\left(e^{a t} E\right) / d t \geqq 0$, and $E(t) \geqq e^{-a t} E(0)$. But

$$
E \leqq B\left(\left\|\dot{q}_{1}\right\|^{2}+\left\|q_{3}\right\|^{2}\right) / 2 \leqq B\left(\|\dot{q}\|^{2}+\left\|q_{3}\right\|^{2}\right) / 2
$$

so

$$
\|\dot{q}\|^{2}+\left\|q_{3}\right\|^{2} \geqq 2 e^{-a t} E(0) / B .
$$

It follows from (17) that $E$ is a nonincreasing function. If $E(0)=0$, then $E$ would vanish identically. It is seen from (21) that this would make $\dot{q}$ vanish identically. This completes the proof.

Theorem 2. Let $V_{1}$ and $V_{3}$ be definite transformations and let $V_{2}$ be a strictly definite transformation. If $g$ is a constant vector, then the differential equation

$$
d V_{1}(\dot{q}) / d t+V_{2}(\dot{q})+V_{3}(q)=g
$$

has a solution such that $\dot{q}=r$, a constant vector. Any other solution is such that $\dot{q}-r$ vanishes exponentially as $t \rightarrow+\infty$.

Proof. Let $Q_{3}$ be the range of $V_{3}$ and now let $Q_{0}$ denote the orthogonal complement of $Q_{3}$. Let $P$ be the orthogonal projection transformation into $Q_{0}$. Then $P$ is a definite matrix such that $P^{2}=P$ and $P Q=Q_{0}$. Let $p=P V_{2}(P q)$, then $d p=P V_{2}^{\prime} P d q$ and $\left(P V_{2}^{\prime} P x, x\right)$ $=\left(V_{2}^{\prime} P x, P x\right)=\left(V_{2}^{\prime} x_{0}, x_{0}\right)$. It follows directly from the definitions of a definite transformation that $P V_{2}(P q)$ is a definite transformation with range $Q_{0}$. By Lemma 4 there is a vector $r$ contained in $Q_{0}$ such 
that $P g=P V_{2}(P r)=P V_{2}(r)$. Thus $V_{2}(r)-g$ is in $Q_{3}$. Applying Lemma 4 to $V_{3}$ shows that there is a vector $s$ contained in $Q_{3}$ such that $V_{3}(s)=-V_{2}(r)+g$.

Let $y=r t+s$. Then $V_{1}(\dot{y})=V_{1}(r), V_{2}(\dot{y})=V_{2}(r)$, and $V_{3}(y)=V_{3}(s)$. Thus $y$ is a solution of (22). If $q$ is another solution, let $z=q-y$. Let $U_{1}(\dot{z})=V_{1}(\dot{q})-V_{1}(\dot{y}), U_{2}(\dot{z})=V_{2}(\dot{q})-V_{2}(\dot{y})$, and $U_{3}(z)=V_{3}(q)-V_{3}(y)$. So

$$
d U_{1}(\dot{z}) / d t+U_{2}(\dot{z})+U_{3}(z)=0 .
$$

From Lemma 7 it is seen that $U_{1}, U_{2}$, and $U_{3}$ are definite transformations. By equation (5), $U_{2}(\dot{z})=U_{2}^{*} \dot{z}$ where $U_{2}^{*}$ is a strictly definite matrix. It is clear that equation (23) is in the form of equation (14). Thus Theorem 1 applies to $z$, and the proof is completed.

ThEOREM 3. Let $V_{2}(\dot{q}, t)$ be a strictly definite transformation as a function of the vector $\dot{q}$ with bounds independent of the parameter $t$. Let $L$ and $S$ be constant definite matrices. In the differential equation

$$
d L \dot{q} / d t+V_{2}(\dot{q}, t)+S q=g(t)
$$

let $g(t)$ be an arbitrary function of $t$. If $q$ and $q^{\prime}$ are both solutions of (24), then $\dot{q}-\dot{q}^{\prime}$ vanishes exponentially as $t \rightarrow+\infty$.

Proof. Subtracting the equations for $q^{\prime}$ and $q$ gives

$$
d L \dot{z} / d t+V_{2}^{*} \dot{z}+S z=0
$$

where $z=q-q^{\prime}$. Here $V_{2}^{*}$ is defined by Lemma 1 . It is seen that Theorem 1 applies to equation (25), and the proof is complete.

4. Application to electrical networks. The network interpretation of the equation $d p_{1} / d t+p_{2}+p_{3}=g$ is as follows. The components of the vectors $p_{1}, p_{2}, p_{3}$, and $g$ give magnetic flux, resistive voltage, capacitative voltage, and generator voltage acting in $n$ independent circuits of the network. The components of $q$ give the net charge which has circulated in these circuits. Thus $\dot{q}$ gives the currents flowing.

The assumption that $p_{1}$ is a definite transformation of $\dot{q}$ corresponds to transformers in the network with ferromagnetic cores. Hysteresis is assumed to be negligible. A detailed analysis of this situation was given in a previous paper [3].

The assumption that $p_{3}$ is a definite transformation corresponds to capacitors having a nonlinear dielectric material. More precisely, the differential dielectric constant should lie between positive limits. Capacitors of this type shall be termed ferroelectric.

In Theorem 1 the matrix $R$ may be interpreted as the effective in- 
stantaneous resistance of the network. The type of nonlinearity permitted is essentially arbitrary. Thus the network could contain vacuum tubes, transistors, or variable resistors. Hysteresis is permitted.

A quasi-linear resistor is a conductor whose differential resistance lies between positive limits. It was shown in a previous paper [1] that if a network has quasi-linear resistors, $p_{2}$ is a definite transformation of the current. In such a situation Theorem 2 applies.

Theorem 2 has the following corollary:

A network of ferromagnetic transformers, ferroelectric capacitors, and quasi-linear resistors powered by batteries cannot generate sustained oscillations or serve as a power amplifier.

The validity of the first part of this statement is clear. In other terms, the statement means that such a network cannot convert direct current to alternating current.

The justification of the second part of the statement depends on the interpretation of the somewhat nebulous phrase "power amplifier." Examples of devices which can serve as power amplifiers are: vacuum tubes, transistors, and relays. This is not the place to give a detailed analysis of the concept of a power amplifier. For the present purpose it suffices to postulate one of the properties of a power amplifier, as follows: If a suitable feedback network is connected between the input and output of a power amplifier, there results a total network with more than one asymptotic state of current flow. The feedback network can be linear, except possibly for quasi-linear resistors. For example, consider an ideal linear power amplifier. Direct positive feedback would result in an unstable system with constant negative resistance in the feedback loop. The adjunction of a suitable quasilinear resistor to the feedback loop would give rise to a system having two stable states of current flow in this loop.

Now consider a network which satisfies the conditions of the corollary. The adjunction of a feedback network results in a total network which satisfies the same conditions. By Theorem 2 the total network has a unique asymptotic state; hence the original network could not have been a power amplifier, and the proof is complete. As stated above, it is assumed that hysteresis is absent. In another paper [4] it is shown that the presence of magnetic or dielectric hysteresis does not change the statement.

Theorem 3 applies to networks with linear transformers, linear capacitors, and quasi-linear resistors. The theorem states that for arbitrary applied generator voltages the effect of the initial state of the network dies out. Thus the current flow approaches a unique asymptotic state as time goes on. This theorem and the theorem 
proved in reference [1] are related but are not equivalent. Either of these theorems has the following corollary:

$A$ network of linear transformers, linear capacitors, and quasi-linear resistors powered by batteries and alternating current generators cannot serve as a power amplifier.

The negation of the statements of these corollaries would have technological significance of some importance. Thus the first corollary implies that the magnetic amplifier or the dielectric amplifier cannot function without a source of alternating current. The second corollary implies that such amplifiers cannot function without the use of nonlinear magnetic or dielectric material.

The writer wishes to acknowledge helpful and suggestive conversations pertaining to the notions in this paper with Professor Leo Finzi of the Department of Electrical Engineering, Carnegie Institute of Technology.

\section{REFERENCES}

1. R. J. Duffin, Nonlinear networks. I, Bull. Amer. Math. Soc. vol. 52 (1946) pp. 833-838.

2. - Nonlinear networks. III, Bull. Amer. Math. Soc. vol. 55 (1949) pp. 119-129.

3. - Nonlinear networks. IV, Proc. Amer. Math. Soc. vol. 1 (1950) pp. 233240.

4. - Impossible behavior of nonlinear networks, Journal of Applied Physics vol. 26 (1955) pp. 603-605.

Carnegie Institute of Technology 